\title{
AXISYMMETRIC POTENTIAL PROBLEMS SUGGESTED BY BIOLOGICAL CONSIDERATIONS ${ }^{1}$
}

\author{
BY R. B. KELMAN
}

Communicated by A. S. Householder, July 15, 1963

1. Introduction. Although axisymmetric potential theory is a welldeveloped subject with many applications to the physical sciences it is, perhaps, not fully appreciated that certain biological problems suggest the use of this theory. In particular the problem of steadystate diffusional flow through a cylindrical structure arises frequently. Not surprisingly, the physiological situation may provide motivation for solving problems and seeking techniques that are different from those arising from purely mathematical or physical considerations. In this paper, which will be reported in full elsewhere, we describe one such problem and outline its resolution.

2. Formulation and solution. The system (1) given below can be regarded as a model for water loss through a plant pore into dry air surrounding the plant. In this model water vapor is the solute and air is the solvent. Let $(r, \theta, x)$ be cylindrical coordinates. Let $a>0$ and $l \geqq 0$ be constants and put $\lambda=l / a$. Put $R_{1}^{0}=\{0<r<a ;-l<x<0\}$, $R_{2}^{0}=\{0<r<a ; x=0\}$ and $R_{3}^{0}=\{r>0 ; x>0\}$ and $R^{0}=R_{1}^{0} \cup R_{2}^{0} \cup R_{3}^{0}$. Let $G(r)$ be a given function such that $r G^{2}(r)$ is Lebesgue integrable on $0 \leqq r \leqq a$. We seek $u(r, x)$ from the system (1) of equations $(n$ denotes outward drawn normal):

$$
\begin{array}{r}
r \frac{\partial^{2} u}{\partial x^{2}}+r \frac{\partial^{2} u}{\partial r^{2}}+\frac{\partial u}{\partial r}=0, \quad(r, x) \in R^{0}, \\
\lim _{\epsilon \rightarrow 0^{+}} \int_{0}^{a}(u(r,-l+\epsilon)-G(r))^{2} r d r=0, \\
u(r, x)=0, \quad x>0 \text { and } r+x=\infty, \\
\frac{\partial u}{\partial n}=0, \quad r=a \text { and }-l<x<0, \\
r>a \text { and } \quad x=0 .
\end{array}
$$

If $\alpha_{k}$ denotes the $k$ th positive root of $J_{1}(r)$ we write the corresponding Dini expansion of $G(r)$ as $G(r) \sim \sum_{0}^{\infty} \mathfrak{g}_{k} g_{0}\left(\alpha_{k} r / a\right)$ where $g_{0}\left(\alpha_{0} r / a\right)$

1 This work was supported in part by the Office of Naval Research, U. S. Navy, under contract Nonr 595(17). 
$=\sqrt{ } 2$ and $J_{0}\left(\alpha_{k} r / a\right)=\sqrt{ }(2) J_{0}\left(\alpha_{k} r / a\right) / J_{0}\left(\alpha_{k}\right)$ when $k=1,2, \cdots$ We denote by $\mathfrak{R}$ the Hilbert space of column vectors $\left\{\mathfrak{r}_{n}: n=0,1, \cdots\right\}$ with square summable components and set $(\mathfrak{x}, \mathfrak{B})=\sum_{0}^{\infty} \mathfrak{r}_{n} \mathfrak{s}_{n}$ and $(\mathfrak{r}, \mathfrak{x})^{1 / 2}=\|\mathfrak{r}\|$. We seek a solution $u$ of $(1)$ and $\mathfrak{i} \in \Re$ such that $u\left(r, 0^{-}\right)$ $\sim \sum_{0}^{\infty} \mathfrak{j}_{k} \mathfrak{J}_{0}\left(\alpha_{k} r / a\right)$.

If $l=0$ and $G=G_{0}$ is a constant, system (1) reduces to Weber's problem of the electrified disk for which it is known that the surfaces of equipotential are oblate spheroids. Therefore in $R_{3}^{0}$ we introduce oblate spheroidal coordinates $(\eta, \phi)$ given by $x=\sinh \eta \cos \phi$ and $r=\cosh \eta \sin \phi$. Separating variables in $R_{3}^{0}$ then suggests seeking $\mathfrak{p} \in \Re$ such that $u\left(r, 0^{+}\right) \sim \sum_{0}^{\infty} \mathfrak{p}_{n}{P_{2 n}}_{2 n}(\mu)$ where $\mu=\left(1-r^{2}\right)^{1 / 2}$ and $\odot_{2 n}(\mu)$ $=(4 n+1)^{1 / 2} P_{2 n}(\mu)$. Thus we are able to write down the formal solution of the problem in terms of $\mathfrak{j}$ and $p$ :

$u(r, x)=\left(\dot{\mathfrak{t}}_{0}+\left(\dot{\mathfrak{j}}_{0}-\mathfrak{g}_{0}\right) \frac{x}{l}\right) \mathfrak{g}_{0}\left(\alpha_{0} r / a\right)$

$$
\begin{array}{cc}
+\sum_{n=1}^{\infty}\left(\frac{\dot{\mathfrak{i}}_{n} \sinh \left(\alpha_{n}(l+x) / a\right)-\mathfrak{g}_{n} \sinh \left(\alpha_{n} x / a\right)}{\sinh \left(\alpha_{n} \lambda\right)}\right) \mathfrak{J}_{0}\left(\alpha_{n} r / a\right), & (r, x) \in R_{1}^{0}, \\
u(\eta, \phi)=\sum_{n=0}^{\infty} \mathfrak{p}_{n} \mathcal{P}_{2 n}(\cos \phi) \frac{Q_{2 n}(i \sinh \eta)}{Q_{2 n}(0)}, & (r, x) \in R_{3}^{0},
\end{array}
$$

where $Q_{2 n}$ is a Legendre function of the second kind. Setting $u\left(r, 0^{-}\right)$ $=u\left(r, 0^{+}\right)$and employing the orthogonal property of the Legendre polynomials yields (in $\Re$ ) a linear algebraic equation $\mathfrak{p}=B \mathfrak{j}$ where $B=\left\{b_{k n}: k, n=0,1, \cdots\right\}$ is a known infinite matrix. Setting $u_{x}\left(r, 0^{+}\right)$ $=u_{x}\left(r, 0^{-}\right)$and employing the orthogonality property of the Bessel functions gives a second equation $\mathrm{i}=A \mathfrak{p}+S \mathfrak{g}$ where $A$ and $S$ are known. Setting $C=A B$ gives the equation $(I-C) \mathfrak{j}=S \mathfrak{g}$ for the unknown $i$. This equation is not symmetric. Symmetrizing it gives the system $\mathfrak{i}=\Lambda^{-1 \mathfrak{l}},(I-D) \mathfrak{l}=T \mathfrak{g}$ where $d_{k k}=0$ and

$$
\begin{array}{r}
\gamma_{k}=\tanh \alpha_{k} \lambda\left[1+\frac{2 \tanh \alpha_{k} \lambda}{\pi \alpha_{k} J_{o}^{2}\left(\alpha_{k}\right)}\left(1+\frac{\sin 2 \alpha_{k}}{2 \alpha_{k}}\right)\right]^{-1}, \quad k=1,2, \cdots, \\
d_{0 n}=-\frac{2}{\sqrt{ } \pi}\left(\frac{4 \lambda \gamma_{n}}{\pi+4 \lambda}\right)^{1 / 2} \frac{\sin \alpha_{n}}{\alpha_{n}^{3 / 2} J_{0}\left(\alpha_{n}\right)}, \quad n=1,2, \cdots, \\
d_{k n}=-\frac{2\left(\gamma_{k} \gamma_{n}\right)^{1 / 2}}{\pi\left(\alpha_{k} \alpha_{n}\right)^{1 / 2} J_{0}\left(\alpha_{k}\right) J_{0}\left(\alpha_{n}\right)}\left[\frac{\sin \left(\alpha_{k}+\alpha_{n}\right)}{\alpha_{k}+\alpha_{n}}+\frac{\sin \left(\alpha_{k}-\alpha_{n}\right)}{\alpha_{k}-\alpha_{n}}\right], \\
k, n=1,2, \ldots ; k \neq n,
\end{array}
$$

and where $\Lambda$ and $T$ are diagonal matrices such that $\lambda_{00}$ 


$$
\begin{aligned}
= & \left(\lambda^{-1}(1+(4 \lambda) / \pi)\right)^{1 / 2}, \lambda_{k k}=\alpha_{k}^{1 / 2} / \gamma_{k}^{1 / 2} \quad(k=1,2, \cdots) \text { and } t_{00} \\
= & (\lambda(1+(4 \lambda) / \pi))^{-1 / 2}, \\
& t_{k k}=\gamma_{k}^{1 / 2}\left[1+\frac{2 \tanh \alpha_{k} \lambda}{\pi \alpha_{k} J_{o}^{2}\left(\alpha_{k}\right)}\left(1+\frac{\sin 2 \alpha_{k}}{2 \alpha_{k}}\right)\right]^{-1}, \quad k=1,2, \cdots .
\end{aligned}
$$

To calculate $\|D\|$, i.e. the least number $M$ such that $\|D \mathfrak{r}\| \leqq M\|\mathfrak{r}\|$ for $\mathfrak{r} \in \Re$, we compare the bilinear form induced by $D$ to the bilinear form induced by the Hilbert matrix $H=\left\{(k+n+1)^{-1}: k, n=0,1, \cdots\right\}$ for which it is known that $\|H\|=\pi$. Direct computation of the bounds for the first 49 elements in $D$ and asymptotic estimates on the remaining elements show that $\|D\|<0.638(0 \leqq \lambda<\infty)$. Thus $\mathfrak{l}=\sum_{0}^{\infty} D^{n} T \mathfrak{g}$ and whence $\mathfrak{i}, \mathfrak{p}$ and $u$ are known. With $\mathfrak{j}$ and $\mathfrak{p}$ thus chosen (2a) and (2b) give a solution harmonic in $R_{1}^{0} \cup R_{3}^{0}$ and satisfying the required boundary conditions. Regularity on $R_{2}^{0}$ is established by showing, mainly using contour integration, that $u$ is a weak solution in $R^{0}$ and then applying Weyl's lemma. From the fact that $I-D$ has a unique inverse it follows that $u$ is unique.

3. Discussion. In physiology interest is centered on the flux, in this case

$$
F=-2 \pi K \int_{0}^{a}\left[\frac{\partial u}{\partial x}\right]_{x=-l}^{r d r}
$$

where $K$ is the diffusion constant, rather than the concentration. Further one is often interested in distinguishing between "active" and "passive" transport whence it is desirable that any formulas derived yield an approximation to $F$, say $\tilde{F}$, such that the relative error, $|\widetilde{F}-F| / F$, is small. The most important case biologically is $G=G_{0}$, e.g. in the case of the plant pore $G_{0}$ is the concentration of saturated water vapor in air. For this case (2a) yields the approximation

$$
\tilde{F}=\frac{4 K G_{0} a}{1+\frac{4 \lambda}{\pi}}\left[1-\frac{0.0449}{1+\frac{4 \lambda}{\pi}}\right]
$$

which gives $F$ with a relative error of less than $5 \%(0 \leqq \lambda<\infty)$ and for the range $\lambda>3.1$ a relative error less than $1 \%$.

It is conjectured that the technique outlined above will be useful in obtaining explicit solutions for other axisymmetric potential problems characterized as follows: the region in question is the union of two regions in each of which the Laplacian is separable. For these two subregions formal solutions can be written in terms of eigenfunc- 
tions appropriate for the regions with the coefficients in the expansions being the unknowns. Algebraic equations in $\Re$ for these coefficients are derived by equating the solutions for the two subregions and their normal derivatives on the interface between the regions.

The two regions can arise from functional as well as geometric considerations. Thus, consider the potential of a cylinder, say $\{0<r<1 ; 0<x<1\}$ where $u=0$ on $\{r=1 ; 0<x<1 / 2\}$ and $\partial u / \partial r=0$ on $\{r=1 ; 1 / 2<x<1\}$, and $u=1$ on $\{0<r<1 ; x=0\}$ and $\{0<r<1 ; x=1\}$. In this case the two regions would be $\{0<r<1$; $0<x<1 / 2\}$ and $\{0<r<1 ; 1 / 2<x<1\}$.

UNIVERSITY OF MARYLAND

\title{
ON LOCAL DIFFEOMORPHISMS ABOUT AN ELEMENTARY FIXED POINT
}

\author{
BY KUO-TSAI CHEN ${ }^{1}$
}

Communicated by Deane Montgomery, July 6, 1963

Let $R^{n}$ be the real $n$-space with $O$ as the origin. Let $G$ be the group of the germs of $C^{\infty}$ local diffeomorphisms about $O$ as a fixed point. We say that $T, T^{\prime} \in G$ are equivalent if they are conjugate in the group $G$. Denote by $\Theta$ the natural homomorphism from the group $G$ onto the group (S) of the $\infty$-jets at $O$. The fixed point $O$ of $T \in G$ will be said to be elementary if the Jacobian $J(T)$ has no eigenvalue of absolute value equal to 1 .

Let $\boldsymbol{A}$ be the Lie algebra (over $R$ ) of the germs of $C^{\infty}$ local vector fields about $O$ and vanishing at $O$. We also use $\Theta$ to denote the natural homomorphism of the Lie algebra $A$ onto the Lie algebra $\mathfrak{A}$ of the $\infty$-jets.

Theorem 1. Let the fixed point $O$ of $U \in G$ be elementary. Then $U$ is equivalent to $T=\phi \eta, \phi, \eta \in G$, such that

(a) $\phi$ is a nonsingular semisimple (i.e., diagonalizable over the field of the complex numbers) linear transformation of $R^{n}$,

(b) $J(\eta)$ is equal to the identity mapping of $R^{n}$ plus a nilpotent linear tranformation,

(c) $\phi \eta=\eta \phi$.

1 This work has been partially supported by the National Science Foundation under Grant NSF-GP-481. 